\title{
1 How to spend a dwindling greenhouse gas budget
} Guido ${ }^{7}$

The Paris Agreement is based on emission scenarios that move from a sluggish phase-out of fossil fuels to large-scale late-century negative emissions. Alternative pathways of early deployment of negative emission technologies need to be considered to ensure that climate targets are reached safely and sustainably.

The historic climate summit in Paris in 2015 galvanized global commitments to an ambitious yet vaguely defined goal of climate stabilization. At the same time some scientists argue that the model-based scenarios with 1.5- and even 2-degree temperature change targets seem unattainable and detached from current political realities ${ }^{1,2}$. Here we scrutinize the dominant climate mitigation scenario archetype that projects low global decarbonization rates in the first half of this century followed by large negative emissions in the second half, thanks to Carbon Dioxide Removal (CDR) technologies ${ }^{3}$. We call this approach to mitigation timing the "Late Century CDR" scenario archetype (Figure 1a). This archetype is consistent with nearly all of 2-degree scenarios covered by the Fifth Assessment Report (AR5) by the Intergovernmental Panel on Climate Change (IPCC) ${ }^{4}, 87 \%$ of which deploy CDR technologies in the second half of the century ${ }^{5}$. Following this predominant archetype might not only turn out to be a risky strategy, but also lead to significant environmental damages and may be economically inefficient. In "Late Century CDR" scenarios, CDR mostly in the form of bioenergy with Carbon Capture and Storage (BECCS) typically removes the equivalent of 20 years of current GHG emissions to reverse the temporary GHG budget overshoot that is tolerated earlier $\mathrm{on}^{6}$. The challenges and uncertainties associated with CDR are well described in the scientific literature ${ }^{5,7}$, yet the scientific and political debate addressing the consequences of large-scale and late deployment of CDR as a "backstop" strategy is only at an early stage. We argue that a new set of scenarios needs to be generated and analyzed to inform the policy process on robust timing of climate mitigation with the aim of avoiding negative side effects. Essentially, three attributes characterize such budget-constrained scenarios: the timing and magnitude of global peak net emissions and its speed of decline thereafter; the maximum amount of allowable deployment of biomass-based CDRs; and an admissible risk threshold associated with a temperature overshoot.

${ }^{1}$ Ecosystems Services and Management Program, International Institute for Applied Systems Analysis (IIASA) Schlossplatz 1, Laxenburg, Austria. ${ }^{2}$ Andlinger Center for Energy and the Environment, Princeton University, Princeton, NJ. ${ }^{3}$ Laboratoire des Sciences du Climat et de l'Environnement, 91191 Gif sur Yvette, France. ${ }^{4}$ Department of Biology, University of Antwerpen, 2610 Wilrijk, Belgium. ${ }^{5} \mathrm{CSIC}$, Global Ecology Unit CREAF-CSIC-UAB, 08193, Cerdanyola del Vallès, Catalonia, Spain. ${ }^{6} \mathrm{CREAF}, 08193$, Cerdanyola del Vallès, Catalonia, Spain. 7 UN Sustainable Development Solutions Network, Paris, France. *e-mail: oberstei@iiasa.ac.at 
A recent climate mitigation assessment has suggested a roadmap for decarbonization consistent with $1.5^{\circ} \mathrm{C}$ warming to be governed by a "carbon law" requiring a 2020 emission peak, halving emissions every decade thereafter and deploying BECCS to the extent of half of today's emissions in $2100^{8}$. We represent this approach in our "Rapid Decarbonization" archetype (Figure 1b). Our calculations confirm that such a carbon law based on a 10 years half-life period could substantially reduce the amount of CDR required (Figure 1b), which is also backed by more complex studies with restricted BECCS deployment and no shortterm mitigation delays ${ }^{9}$. However, although desirable, rapid emission reductions face some real world challenges, including inertia in the energy system, failure to coordinate mitigation targets at global or national level, or upward trends in emissions from non-point and non-CO2 GHG sources - all of which underpin the rationale for CDR.

More sophisticated modelling approaches incorporate such challenges and yield less optimistic fossil decarbonization rates. For example the scenarios combining Shared Socioeconomic Pathways (SSP) and Representative Concentration Pathways $(\mathrm{RCP})^{3}$ span a range from relatively fast mitigation (e.g. SSP1) to scenarios with a delayed response. Depending on the storyline underlying their levels of mitigation challenges the $2^{\circ} \mathrm{C}$ compatible scenarios are characterized by half-life periods of 20 years and more. Higher obstacles to fossil decarbonization take place at the cost of potentially large-scale deployment of BECCS in the late $21^{\text {st }}$ century (see Supplementary Figures 1 and 2). More than $85 \%$ of these scenarios show maximum BECCS capacity in 2100 and primary bioenergy supplying an amount equivalent to roughly $80 \%$ of current total primary energy demand. In currently prevailing climate mitigation scenarios from the IPCC AR5 for the 1.5- and 2-degree targets BECCS is peaking at colossal rates of 8-20 Gt CO2 per year at the end of the century. Another strong assumption underlying a large share of these scenarios (and more than $80 \%$ of the RCP2.6 scenarios) is that net carbon emissions peak in 2020. A later peak of net emissions leads to even higher BECCS deployment at the end of the century, shown in Figure 2.

\section{Late peak BECCS}

For a number of reasons late century peak BECCS is problematic. The large scale deployment of BECCS might turn out to be environmentally and socially damaging and thereby not be consistent with the very objective of the UNFCCC and the sustainable development goals. Depending on the specific scenario roughly between 200 and 1100 million ha (SSP2) and up to 1500 million ha (SSP4) ${ }^{10}$ amounting to almost all of current global cropland area ${ }^{11}$ are expected to be allocated to energy crop in the RCP2.6 scenarios, with a largely unknown carbon debt and large-scale impacts on ecosystems functions such as biodiversity, water and nutrient cycling, and regional climate attenuation. Most of the damaging conversion is planned to happen in the last three decades before 2100 , at a time when the pressure on land-based natural capital assets is likely to be high, but still difficult to assess based on current drivers. Furthermore, land-based mitigation in combination with BECCS might have a strong impact on food prices which could be associated with food security even later in the century ${ }^{12,13}$.

End of century peak BECCS would lead to large scale stranded assets. BECCS is associated with building significant amounts of fixed technical capital in terms of large-scale plantations, biomass transportation infrastructures, geological storages, CO2 pipelines and CCS installations. Late century peak of BECCS capacity once the temperature change target is reached in 2100 lead to a situation where BECCS is no 
longer needed in the year 2101 unless in the UNFCCC at its 100 year anniversary countries jointly decide to go for an even lower temperature change target for the $22^{\text {nd }}$ century.

Late century peak BECCS is also a consequence of an overshoot in cumulative emissions, which may be associated with feedback effects from the earth system ${ }^{14}$, both with the risk of passing a dangerous temperature threshold (e.g. ice sheet melting, thawing of permafrost and/or feedbacks from the carbon cycle induced by other $\mathrm{GHGs})^{15}$ and with the well-known behavior of the carbon cycle that if $\mathrm{CO}_{2}$ decreases at a steep rate, the ocean and natural ecosystems will switch from sink to sources ${ }^{16}$.

Finally, late-peak BECCS (or other CDRs, such as Direct Air Capture (DAC) or Enhanced Weathering) means that we substantially rely on technologies that are still in their infancy ${ }^{17,5}$ and whose risks under largescale deployment have not been explored fully, or may prove not be scalable.

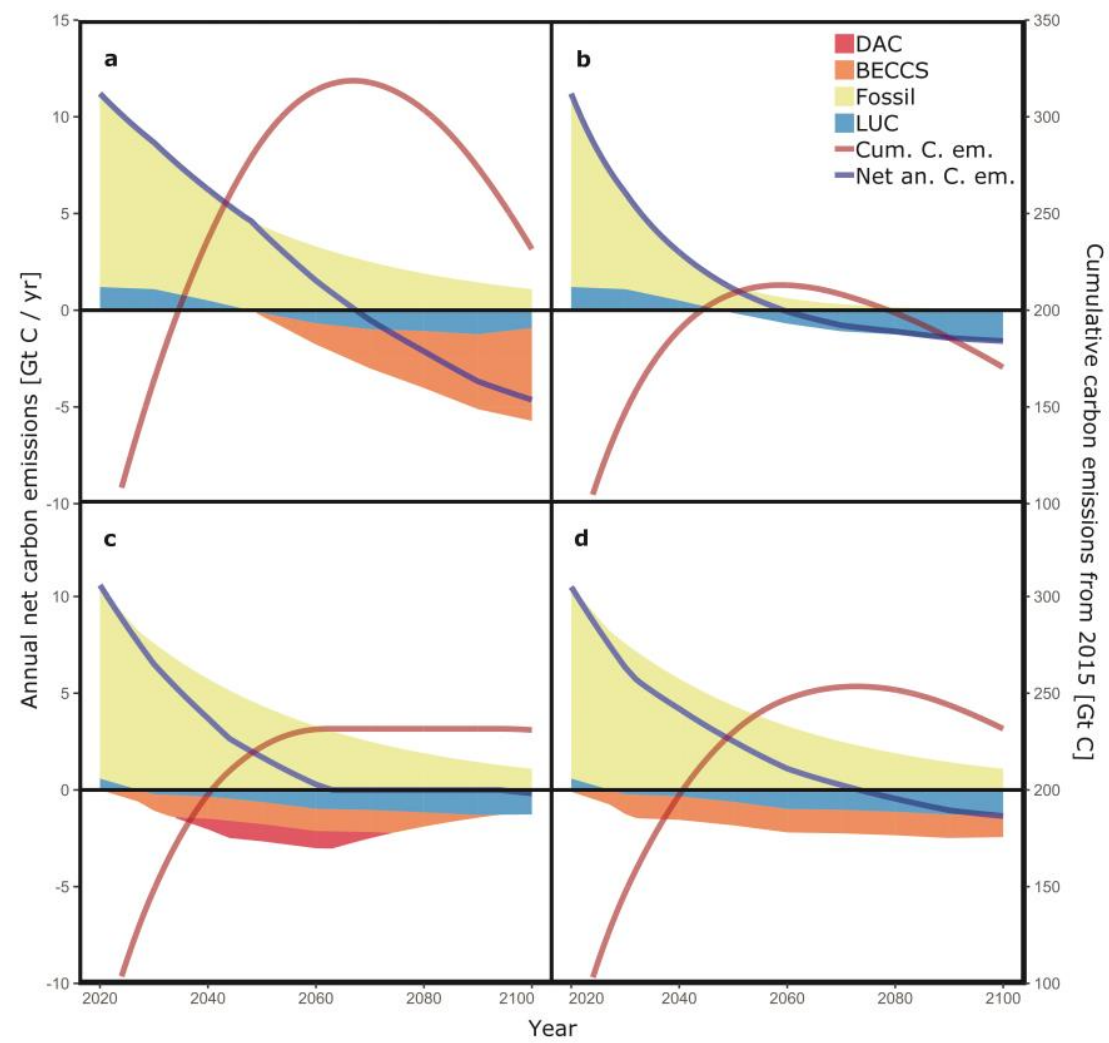

Figure 1: Four archetypes of emission pathways leading to a $2^{\circ} \mathrm{C}$ warming target with peak emissions in 2020. Projections are based on a "threshold exceedance budget" of $232 \mathrm{Gt}$ C for 2015-2100 including an RCP8.5 non-CO2 forcing ${ }^{23}$. Blue lines depict annual net $\mathrm{C}$ emissions, red lines are cumulative $\mathrm{C}$ emissions. a) "Late Century CDR": Late century BECCS deployment results in a substantial overshoot of cumulative emissions (half-life of fossil phase-out $=25$ years); b) "Rapid Decarbonization": A "carbon law" with 10 years half-life makes CDR obsolete since cumulative emissions stay well below the budget; c) "No Overshoot": CDRs ramp up early and phase out towards the end of the century to avoid an overshoot in cumulative emissions, BECCS is limited to 1.2 Gt C/yr (equaling the BECCS-capacity of the "Minimize CDR" archetype), the remainder is captured by DAC (half-life of fossil phase-out $=25$ years); d) "Minimize CDR": BECCS is spread evenly over the century thereby minimizing its capacity. No other CDRs are deployed (half-life of fossil phase-out $=25$ years). AFOLU-sector C-price for $a$ ) and $b$ ) increases exponentially from 0 (in 2020) to 200 (in 2100), for c) and d) it is constant at 40 USD2000 per t CO2. 


\section{Why early CDR?}

The arguments above point to the undesirability of the currently dominant "Late Century CDR" climate mitigation archetype. However, early deployment of BECCS in deterministic and perfect foresight scenarios does not occur due to discounting over a 100 year time horizon. Discounting in conjunction with a limited carbon budget induces an exponentially increasing carbon price ${ }^{18}$, reflecting a time preference for deferring investments at typically $5 \%$ per annum.

There are a number of compelling reasons for early deployment, and thus substantially reduced peak deployment, of CDR. In fact, the original concept of CDR deployment was framed in a climate risk management framework with anticipative implementation of recarbonization measures of landscapes, optionally to be augmented by BECCS and other forms of long-term carbon storage later, if climate change risk signals become eminent ${ }^{19}$. Also in cases where an overshoot is found to be too risky from a climate science perspective, deployment of early CDR becomes more valuable ${ }^{20}$. Finally there is the argument of intergenerational equity to carry the burden of mitigation efforts. Early decarbonization (Figure 1b) of the fossil sector will minimize or avoid altogether the need to deploy engineered CDR technologies of potentially high economic and environmental costs to be incurred by a generation which is just being born. For mitigation pathways where engineered CDR is unavoidable (e.g. Paris agreement) the application of an intergenerational equity principle would suggest to spread the deployment of engineered CDR more evenly, but at much smaller deployment rates, within the $21^{\text {st }}$ century.

\section{Alternative archetypes}

In addition to the existing "Late Century CDR" archetype and the recently introduced "Rapid Decarbonization" approach, we suggest the production of new scenarios along alternative archetypes. These archetypes are characterized by early deployment of mostly biological and terrestrial CDR, which might deliver important ecosystem services by recarbonizing landscapes. We illustrate all archetypes of climate mitigation pathways in Figure 1 and benchmark these in Figure 2. Moreover, we quantify the value of early action with respect to mitigation by comparing "peak 2020" archetypes to the same scenarios where only the peak of net emissions is delayed from 2020 to 2025 followed by a "carbon law" for fossil emission phase-out effective from 2030 (see Supplementary Figure 3). For a detailed discussion on the construction of these scenarios see Supplementary Information.

The "No Overshoot" archetype (Figure 1c) avoids exceedance of the cumulative emissions budget by early introduction of CDR. This archetype is most conservative with respect to the need to resort to a CDR backstop later in the century. The "Minimize CDR" archetype is about early deployment and ramping-up BECCS to an allowable maximum to be maintained throughout the century (Figure 1d). This strategy minimizes the peak CDR capacity, but still has an overshoot in cumulative emissions. These two new archetypes show similar early BECCS deployment, resulting in an option to choose between two alternative pathways between 2030 and 2040. At that point in time new findings from climate science and technological innovation could be incorporated to refine the negative emission strategy (e.g. deployment of DAC instead of BECCS). Like the majority of RCP2.6 scenarios (Supplementary Figure 1), the "Late Century CDR" archetype as well as the two new archetypes are based on half-life periods of 25 years, while the "Rapid Decarbonization" type is characterized by a 10 year "carbon law". 
In Figure 2, the level of performance of all archetypes in each benchmark category is visually supported by a color gradient from green (good performance) to red (bad performance). The discounted cost minimizing "Late Century CDR" archetype is outperformed in each of the selected benchmark categories. "No overshoot" minimizes stranded assets and the risk associated with temperature overshoots at potentially large near-term costs for early and large scale CDR (including DAC). "Minimize CDR" represents a trade-off between benchmark performance and necessary investments to achieve moderate levels of CDR. "Rapid decarbonization", if applied early, could essentially make CDR obsolete. However, if delayed, its environmental and socio-economic impact depends on the CDR strategy at hand. Combined with end of century BECCS, as proposed by Rockström et al. ${ }^{8}$ it would lead to an essential increase in the overshoot level and potential stranded assets. The effect of delaying peak annual net emissions for only 5 years is striking for all of the archetypes leading to extreme figures especially for the "Late Century CDR" archetype, such as an alarming overshoot level of $116 \mathrm{Gt} \mathrm{C}$ and a potential natural land loss of 33\% compared to year 2000 levels.

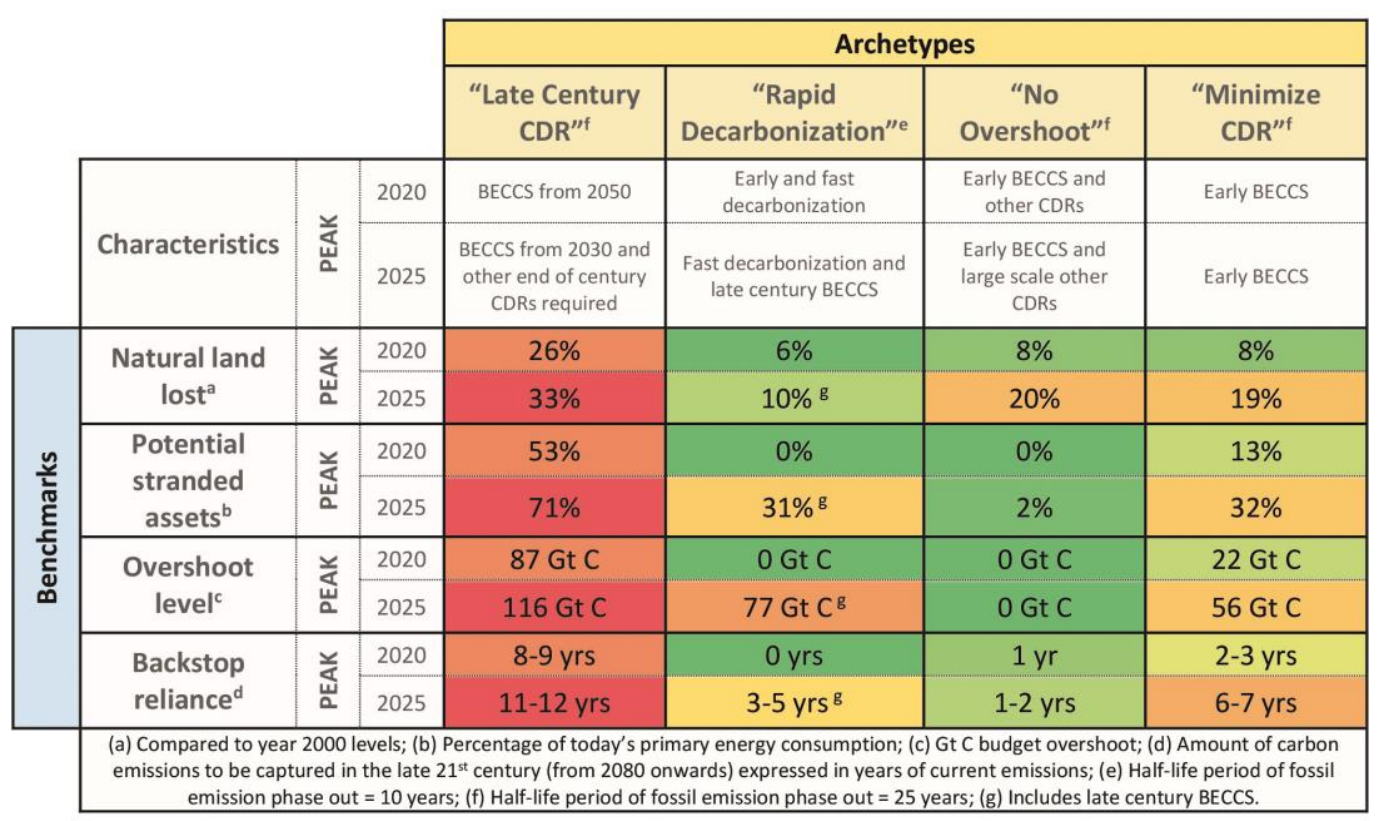

Figure 2: The new archetypes "Minimize CDR" and "No Overshoot" are benchmarked against prevailing archetypes "Rapid Decarbonization" and "Late Century CDR" based on results from our own model calculations presented in the supplementary material. The level of performance is visually supported by a color gradient from green (good performance) to red (bad performance). Different time horizons for the peak of net carbon emissions illustrate the value of early action. Peak 2020 is illustrated in Figure 1, peak 2025 in Supplementary Figure 3. The prevalent "Late Century CDR" archetype, if delayed, would require BECCS to be initiated not long after 2030, culminating in a $116 \mathrm{Gt}$ C cumulative emission overshoot, thereby potentially creating stranded assets to the extent of roughly $2 / 3$ of our present primary energy consumption and requiring CDR technologies to recapture the equivalent of more than 10 years of present emissions between 2080 and 2100 . Only the undelayed "Rapid Decarbonization" scenario as well as the "No Overshoot" scenario are characterized by lower BECCS capacities in the second half of the century, the latter relying on heavy deployment of other negative emissions technologies, if mitigation is delayed. 


\section{Conclusion}

2 We conclude that the timing of mitigation actions, in particular of negative emission technologies, needs 3 to be urgently revisited in the analyses of ambitious climate targets. We argue that considerations of both 4 intergenerational equity and climate/environment safety motivate early and moderate -- rather than 5 extreme -- deployment of negative emission technologies as well as a timely peak in net carbon emissions 6 as early as 2020. As a consequence all of the near-term and mid-century net emission reduction targets 7 should be reformulated including targets of early action on CDR technology portfolios. Furthermore, our 8 calculations point to significant indirect land use effects and other cascading impacts of delayed actions 9 in phasing out fossil fuel emissions. There is an inter-temporal substitution between sluggish fossil fuel 10 emissions today and undesirable land use and food system impacts later. Policy assessments informing 11 near-term technology preferences should therefore account for such lagged environmental and social 12 external costs.

13 Yet, early development of CDRs will be associated with significant policy challenges as witnessed by the 14 debates around biofuels ${ }^{21}$, avoided deforestation and forest carbon sequestration ${ }^{22}$. Transforming the 570 15 million farms to be climate smart and incentivizing 1.6 billion people who economically depend on forests 16 to become early movers in "No overshoot" and "Minimize CDR" scenarios is a formidable global policy 17 challenge. We call for a discourse on effective strategies, starting with more detailed global gap 18 assessments of the archetypes, and then mainstreaming the gained insights into Nationally Determined 19 Contributions (NDCs) and implementation plans. 
1

2 Acknowledgement

3 The authors would like to acknowledge the financial support from the European Research Council Synergy 4 grant ERC-SyG-2013-610028 IMBALANCE-P. 
2 1. Tollefson, J. Nat. News 527, 436 (2015).

3 2. Raftery, A. E., Zimmer, A., Frierson, D. M. W., Startz, R. \& Liu, P. Nat. Clim. Change advance online 4 publication, (2017).

5 3. Riahi, K. et al. Glob. Environ. Change 42, 153-168 (2017).

$6 \quad$ 4. Clarke, L. (2014).

7 5. Smith, P. et al. Nat. Clim. Change 6, 42-50 (2016).

8 6. IPCC, 2014 [Core Writing Team, R.K. Pachauri and L.A. Meyer (eds.)]. IPCC, Geneva, Switzerland, 151 $9 \quad \mathrm{pp}$.

10 7. Fuss, S. et al. Nat. Clim. Change 4, 850-853 (2014).

11 8. Rockström, J. et al. Science 355, 1269-1271 (2017).

12 9. Riahi, K. et al. Technol. Forecast. Soc. Change 90, 8-23 (2015).

13 10. Popp, A. et al. Glob. Environ. Change 42, 331-345 (2017).

14 11. Fritz, S. et al. Glob. Change Biol. 21, 1980-1992 (2015).

15 12. Obersteiner, M. et al. Sci. Adv. 2, e1501499 (2016).

16 13. Havlik, P. et al. in Climate Change and Food Systems: Global assessments and implications for food 17 security and trade (ed. Elbehri, A.) 178-197 (FAO, 2015).

18 14. Allen, M. R. Nat. Clim. Change 6, 684-686 (2016).

19 15. MacDougall, A. H., Zickfeld, K., Knutti, R. \& Matthews, H. D. Environ. Res. Lett. 10, 125003 (2015).

20 16. Peñuelas, J. et al. Nat. Ecol. Evol. 1, 1438-1445 (2017).

21 17. National Research Council, Division on Earth and Life Studies, Ocean Studies Board, Board on

22 Atmospheric Sciences and Climate \& Impacts, C. on G. C. T. E. and D. of. (National Academies Press, 23 2015).

24 18. Hotelling, H. J. Polit. Econ. 39, 137-175 (1931). 
1 19. Obersteiner, M. et al. Science 294, 786-787 (2001).

2 20. Lemoine, D. M., Fuss, S., Szolgayova, J., Obersteiner, M. \& Kammen, D. M. Clim. Change 113, 141$3162(2012)$.

4 21. Searchinger, T. D. et al. Science 326, 527-528 (2009).

5 22. Gren, I.-M. \& Aklilu, A. Z. For. Policy Econ. 70, 128-136 (2016).

6 23. Rogelj, J. et al. Nat. Clim. Change 6, 245-252 (2016). 